\title{
BMJ Open Policy environment impacting the societal harm caused by alcohol in India: protocol for a scoping review
}

\author{
Jaclyn Schess, ${ }^{1,2}$ Abhijeet Jambhale, ${ }^{2}$ Urvita Bhatia, ${ }^{2}$ Richard Velleman, ${ }^{2,3}$ \\ Abhijit Nadkarni ${ }^{2,4}$
}

To cite: Schess J, Jambhale A, Bhatia U, et al. Policy environment impacting the societal harm caused by alcohol in India: protocol for a scoping review. BMJ Open 2018;8:e020854. doi:10.1136/ bmjopen-2017-020854

- Prepublication history and additional material for this paper are available online. To view these files, please visit the journal online (http://dx.doi. org/10.1136/bmjopen-2017020854).

Received 27 November 2017 Revised 17 September 2018 Accepted 11 October 2018

Check for updates

(c) Author(s) (or their employer(s)) 2018. Re-use permitted under CC BY-NC. No commercial re-use. See rights and permissions. Published by BMJ.

${ }^{1}$ Yale University, New Haven, Connecticut, USA

${ }^{2}$ Addictions Research Group, Sangath, Porvorim, Goa, India ${ }^{3}$ Department of Psychology, University of Bath, Bath, UK ${ }^{4}$ Department of Population Health, London School of Hygiene and Tropical Medicine, London, UK

Correspondence to Professor Richard Velleman; r.d.b.velleman@bath.ac.uk

\section{ABSTRACT}

Introduction The aim of this review is to provide the first consolidation of the policy environment surrounding alcohol-related societal harm in India giving researchers and policy-makers a clearer base for future reforms. This review is also an important adaptation on the scoping review method for policy reviews in low-resource settings that may serve as an example for other policy reviews in similar settings.

Methods and analysis We will undertake a scoping review with policy relevant adaptations in order to map the alcohol-related policy environment in India. Following the six-step approach put forward by Arskeyand0'Malley and refined by Levac, we will first undertake an academic scoping search to identify relevant knowledge already existing in the literature about the policy environment in India. We will then use the knowledge that appears in this search iteratively, as is true to the scoping method, to develop a more targeted search of grey literature and Indian government websites for Indian policy documents. These documents will be analysed using qualitative methods to synthesise the current alcohol policy environment in India.

Ethics and dissemination This study will only use already published information and therefore does not require an ethics review. We will circulate this protocol and the final report to policy researchers in similar settings who could make use of our adaptation of the scoping review method for a low-resource setting. We will also publish our findings in a peer-review journal.

\section{INTRODUCTION}

Alcohol consumption is a cause of substantial preventable societal harm in Southeast Asia. In 2010, the prevalence of alcohol use disorders (AUDs) in southeast Asia was 2.2\%. The alcohol attributable burden of disease accounted for an average $2.7 \%$ ofDisability Adjusted Life Years (DALYs) in 2004 in southeast Asia, ${ }^{2}$ with prevalence continuing to rise in the region. ${ }^{3}$ In addition to the burden of ill health, alcohol consumption creates significant societal economic costs, equal to more than $1 \%$ of gross domestic product (GDP) in high-income and middle-income countries, ${ }^{2}$ through increased health costs and decreased

\section{Strengths and limitations of this study}

- This is a novel review approach to reviewing a policy environment in a low-resource setting where relevant policies can be difficult to identify through traditional search methods.

- This is the first review of its kind to attempt to consolidate the policy environment applicable to the societal harm related to alcohol in India.

> Although the sources cited are dated they are the most recent, further emphasising the necessity of investigating the research gap.

- This review seeks to investigate a policy area that is extensive in scope, and though this may limit the ability of the search to explore deeply every policy area related to the societal harm caused by alcohol in India, the review's intention is to identify and analyse, at the very minimum, existing key policies related to this topic.

- The search is limited to texts in English.

economic productivity. In India, alcohol use contributed $5.4 \%$ of the total burden of disease in 2004, significantly higher than the region average. ${ }^{2}$ By 2014, this estimate was up to $8.8 \%$, connected to 1.4 million cases of 19 diseases. ${ }^{4}$ In addition, consumption of alcohol has been steadily increasing in India, with recorded per capita liquor consumption increasing from $1.6 \mathrm{~L}$ in $2003-2005$ to $2.2 \mathrm{~L}$ in 2010 to $4.3 \mathrm{~L}$ in $2014,{ }^{4}$ and more than half of all drinkers are drinking hazardously. ${ }^{5}$ Total alcohol consumption is projected to reach 4.7 L per capita by $2020 .^{6}$

This paper will discuss societal harm caused by alcohol, defined as alcohol-related harm to the drinkers themselves and also the harmful consequences of drinking to those around the drinker-externalities of drinkingan element which receives very little policy focus. $^{78}$ As demonstrated in India, about a quarter of abuse against women and children has been connected to chronic alcohol use. ${ }^{9}$ In addition, approximately one-third of night-time road traffic injuries and mortalities 
involved a driver who had consumed alcohol. ${ }^{9}$ Further, alcohol use contributes to homelessness, ${ }^{10}$ absenteeism and unemployment, ${ }^{11}$ high-risk sexual behaviour ${ }^{12}$ and poor health outcomes for children. ${ }^{13}$ Finally, the yearly overall alcohol use-related economic costs to society in India were estimated to be 2.1 trillion Indian rupees (US $\$ 30.8$ billion) in 2013-2014, or roughly $2.26 \%$ of the GDP. ${ }^{414}$

While there is growing recognition of the preventable harm that alcohol use is leading to in India, the country still lacks a consolidated national alcohol policy. This protocol will define policy as done by the USA's Centers for Disease Control: 'Policy is a law, regulation, procedure, administrative action, incentive, or voluntary practice of governments and other institutions'. ${ }^{15}$ This lack of consolidated policy is evident in the focus on prohibition-centred supply reduction, taxation and tertiary prevention ${ }^{5}$; all implemented as a set of disparate and poorly coordinated policy initiatives. An additional concern is the power of the 'alcohol lobby' and especially of multinational alcohol producer and distributor corporations, that view India's population as an emerging market of new and young drinkers, who, over time, are continuing to increase their consumption and demonstrate a reducing age of initiation; all of which make a national policy even timelier. ${ }^{16}$ India is a key example of WHO Director General Dr Margaret Chan's warning: "Alcohol consumption is expanding in precisely those countries that lack the regulatory and enforcement capacities to protect their populations."17

Given the absence of a comprehensive national alcohol policy, we believe there is a necessity to comprehensively map the current policy environment surrounding alcohol and its use in India, something that has not been undertaken in the existing literature, and this is the goal of the review described herein. A comprehensive mapping would allow for the examination of these policies, helping researchers and practitioners better understand the exact policy environment, or the total composition of all the policies in place, with which alcohol-related societal harm is interacting in India. This can also help policy-makers, those who are involved in any extent in making or changing policies in place, get a clearer and more comprehensive picture of the current state of alcohol-related policies across India, in order to take steps towards reform. In performing this mapping, this protocol also puts forward an important adaptation of the scoping review method for use for policy research in low-resource settings.

\section{METHODS}

The methods for this review use the scoping review methodological framework put forward by Arskey and $\mathrm{O}^{\prime}$ Malley ${ }^{18}$ and refined by Levac et al. ${ }^{19} \mathrm{~A}$ scoping review, as compared with a systematic review, uses a six-stage process of review and allows for covering of broad topics of research areas and iterative construction of research direction. Where systematic reviews have strictly defined questions and therefore eligibility criteria at the outset, scoping reviews are iterative in nature ${ }^{19}$ and eligibility parameters and search strategies are therefore refined as the search identifies more relevant knowledge.

We considered the various methods available for conducting a policy review and decided that a scoping review with relevant adaptations would be the most applicable to reviewing intersectorial policy in an Indian context. Our strategy will start with an academic search of peer-reviewed literature to iteratively define search parameters for grey literature and government website searches. In doing this, our search adapts the scoping review method by adopting a tiered approach, an 'academic search tier' followed by a 'grey literature search tier' in order to identify policy documents. The detailed steps of our search strategy are as follows:

\section{Stage 1: identifying the research question(s)}

Based on an iterative review of the literature and the previous experience of the investigator group in examining the burden of AUDs in India and developing interventions to reduce the harm due to AUDs, ${ }^{20-23}$ our guiding research questions were developed. Our objective is to comprehensively map Indian policies that relate to alcohol-related societal harm and frame these in the context of international standards for alcohol policy. More specifically, we ask the following questions:

What are the policies currently in place in India that regulate demand for and supply of alcohol and alcohol products?

What are the policies currently in place in India that regulate the impact on a range of societal outcomes arising from access to, use and misuse of alcohol?

This includes AUDs, as well as drink-driving, violence, microeconomic and macroeconomic outcomes, comorbid health outcomes, family member health outcomes and any other negative impacts which alcohol has on Indian society. Our questions and scope remain broad in nature given the lack of current research and disparate policy environment expected in India where there is no national alcohol policy. We therefore leave our scope to include policies which may not be specific to alcohol but which include components that relate to alcohol use.

\section{Stage 2: identifying relevant studies}

The first tier of our study involves identifying relevant academic literature that discuss policies which are relevant to our final search. In line with the iterative process of a scoping review, this will help set the search terms for the grey literature search for policy documents, using knowledge generated from the peer-review literature. For a description of the objectives and inclusion and exclusion criteria at each tier of the search, see figure 1 .

Electronic databases of peer-reviewed journals MEDLINE, PSYCInfo, Embase, Global Health and IndMed (database of peer-reviewed medical journals published from India) were searched in August and 


\section{Tier 1: Academic Search}

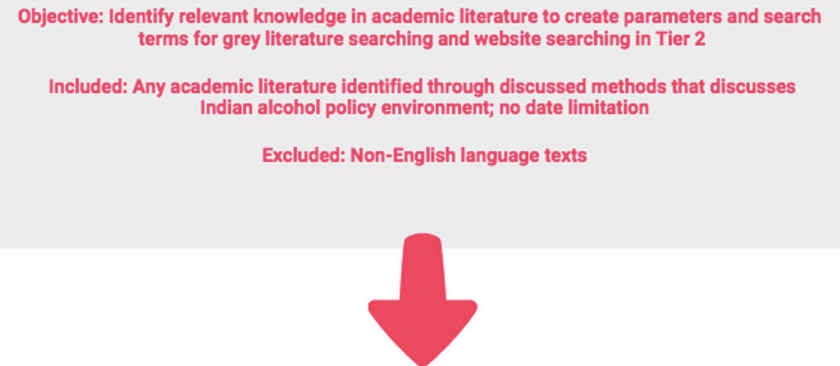

Tier 2: Policy Search

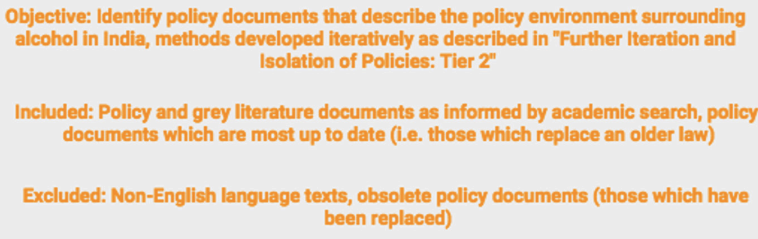

Figure 1 'Academic search tier' as it informs 'Policy search tier' including descriptions of the objective, inclusion and exclusion criteria of each.

September 2017. All search hits were downloaded to a bibliographical management software. Search terms used centred on the concepts of 'alcohol', 'policy' and 'India'. The 'alcohol' concept was built around synonyms and types of alcohol (eg, spirit, liquor, beer, illicit alcohol) and terms applying to drinking (eg, drinker, alcohol consumption, social drinking, bars) and alcohol misuse (eg, addiction, problematic alcohol use, alcohol dependence, drink driving). The 'policy' concept was broad and included terms covering all dimensions of policy applicable to alcohol including marketing, sales, production, importation, trade, treatment and synonyms for these terms (eg, deaddiction, promotion, taxes, availability, minimum drinking age, prohibition, labelling, prevention, lobbying, industry and many others). The 'India' concept included terms for India (eg, Indian Union) and all of its states and union territories including historical state names. The search terms under these three concepts were used to search the databases mentioned above using a title/abstract/keyword search as well as a subject heading search where possible. The full search strategy can be found in online supplementary file 1 .

\section{Stage 3: study selection}

Two reviewers (JS and AJ) will independently screen titles and abstracts resulting from the search process and exclude any ineligible studies. This process is ongoing at the time of the protocol submission. The researchers will discuss their selection of articles after this first round of screening, to arrive at preliminary consensus on the list of eligible studies. They will then complete a full-text screening of all selected articles, removing any articles that do not meet the inclusion criteria, as abstracts contain less detail than the full text. Reviewers will again discuss their selection of articles after this second round of screening, to arrive at a final consensus list of eligible studies. Differences in opinion will be resolved through consensus between the two reviewers with a third reviewer (AN) serving as arbiter in case of disagreement.

At both levels, the reviewers will use the following questions for guiding inclusion and must answer yes to all three questions to include an article: (1) Does this article discuss specific policies? (2) Does this article discuss specific policies relating to alcohol or alcohol's externalities? (3) Does this article discuss policy specific to India?

This systematic process will be reported using the Preferred Reporting Items for Systematic Reviews and Meta-Analyses flow chart. ${ }^{24}$

\section{Stage 4: charting the data}

Data extraction forms will be created and used to chart relevant data from included studies. Two researchers (JS and $\mathrm{AJ}$ ) will independently extract data. Data extraction will be compared after the first 10 documents to check for consistency between the two researchers and allow for form revision, and then again, after data extraction from all documents. Data extraction at this stage will involve collecting knowledge about policy that will be used iteratively to find policy documents in tier 2 of the search. Data to be charted may include but not be limited to title of publication, year of publication, policy name, policy description, implementing jurisdiction and date/year of policy implementation.

\section{Stage 5: collating, summarising and reporting results}

Data from the academic search will be summarised into a list of policy names, a list of possible search terms and a list of potentially relevant citations to follow-up. The research team will then discuss the list and make decisions for further iterative search processes of grey literature, government websites and other strategies to find policy documents. For further analysis, the policies will be further mapped according to the WHO's Global Strategy to Reduce the Harmful Use of Alcohol which includes 10 strategy areas across different policy sectors. ${ }^{25}$

\section{Further iteration and isolation of policies: tier 2}

The peer-review tier of our search will help generate knowledge of the parameters in which to search the grey literature and government websites. Scanning of citations, isolating of policy names and descriptions, and knowledge gathering of relevant vocabulary will all be used to iteratively develop the strategies to isolate policies.

We will then consult with leading researchers and policy-makers based on the collated data from tier 1 of the search to gain insight and scrutiny of our next steps for searching the grey literature in order to ensure that we are not missing any important documents in tier 2. 
These policy documents will be the data to be included in tier 2 with planned coverage in September-November 2018. Following an iterative approach, we will use the knowledge generated from tier 1 in combination with researcher knowledge to help identify strategies for searching for policy documents. Possible strategies to subsequently identify and gather policy documents include the searching of grey literature through WHO and World Bank websites and use of Google and/or Google Scholar. Google and English language websites of state and national Ministry websites, which are found to be relevant during our academic search, will be handsearched for policies. As more knowledge is obtained through this process, we will continue to iteratively search through grey literature. Through these steps, we will aim to obtain the key policy documents that create the policy environment relating to alcohol-related societal harm.

Tier 2 data will then be charted using a data extraction form. This form will aim to gather data relating to policy name, department responsible, goal of policy and other details, but will be developed by the team after the iterative grey literature stage has been undertaken to ensure the chart is most useful. The developed data chart will be piloted on 5-10 policy documents to ensure its usefulness once developed, and changes will be made accordingly.

Data from the charting of tier 2 will be synthesised, summarised, discussed within the team and compared with what was originally found in the academic literature. We will analyse the data with a thematic analysis approach to present the themes that emerge from the policies present. This is in line with the scoping review approach which aims to present an overview of a research area instead of appraising the quality of data. ${ }^{18} 19$ Data will be further mapped according to the WHO Global Strategy. This will allow for global comparisons and identification of gaps in policy according to the recommendations of the WHO in the Global Strategy for best practices in reducing the harm of alcohol.

\section{Stage 6: consultation}

We will consult with leading researchers and policy-makers in India, on our consolidated list of policies and the thematic analysis and mapping of these policies to ensure that we have not missed any crucial policies in our search.

\section{Patients and public involvement}

No patients are involved in this review.

\section{ETHICS AND DISSEMINATION}

The study was submitted for registration on PROSPERO, but investigators were informed that scoping studies are not in scope for registration. We will be circulating the methods used for this policy review to other policy researchers, particularly those researching in low-resource settings, as we believe this method is helpful in navigating intersectorial policy spaces in low-resource settings. We will publish our results in a peer-reviewed journal and on relevant forums such as online global health networks.

Contributors All authors have made substantial intellectual contributions to the development of this protocol and its revisions. The search question was conceptualised by AN and further developed by RV. The review approach and design was conceptualised by JS with advice from AN. JS and AJ developed and tested search terms with input and revisions from AN, RV and UB. JS initiated drafting of the manuscript followed by further iterations after substantial input and appraisal from all authors. All authors approved the final version of this manuscript.

Funding JS was supported by funding from the Yale University Jackson Institute of Foreign Affairs and the Yale University MacMillan Center for International and Area Studies.

Competing interests None declared.

Patient consent Not required.

Ethics approval This scoping study does not require ethics approval as it only uses secondary data.

Provenance and peer review Not commissioned; externally peer reviewed.

Open access This is an open access article distributed in accordance with the Creative Commons Attribution Non Commercial (CC BY-NC 4.0) license, which permits others to distribute, remix, adapt, build upon this work non-commercially, and license their derivative works on different terms, provided the original work is properly cited, appropriate credit is given, any changes made indicated, and the use is non-commercial. See: http://creativecommons.org/licenses/by-nc/4.0/.

\section{REFERENCES}

1. World Health Organization. Global Information Systems on Alcohol and Health (GISAH). 2018. http://www.who.int/gho/alcohol/en/

2. Rehm J, Mathers C, Popova S, et al. Global burden of disease and injury and economic cost attributable to alcohol use and alcohol-use disorders. Lancet 2009;373:2223-33.

3. Whiteford HA, Degenhardt L, Rehm J, et al. Global burden of disease attributable to mental and substance use disorders: findings from the Global Burden of Disease Study 2010. The Lancet 2013;382:1575-86.

4. WHO Country Office for India. Assessment of burden and socioeconomic cost attributable to alcohol use in India. 2014. http://www.searo.who.int/india/publications/assessment_burden_ socioeconomic_cost.pdf

5. Benegal V. India: alcohol and public health. Addiction 2005;100:1051-6.

6. World Health Organization. Total consumption, projections to 2025 by country. 2016. http://apps.who.int/gho/data/node.main.A1043? lang=en

7. Greenfield TK, Ye Y, Kerr W, et al. Externalities from alcohol consumption in the 2005 US National Alcohol Survey: implications for policy. Int J Environ Res Public Health 2009;6:3205-24.

8. Room R, Ferris J, Laslett AM, et al. The drinker's effect on the social environment: a conceptual framework for studying alcohol's harm to others. Int J Environ Res Public Health 2010;7:1855-71.

9. Gururaj G, Murthy P, Girish N, et al. Alcohol related harm: Implications for public health and policy in India. 2011. http:// nimhans.ac.in/cam/sites/default/files/Publications/39.pdf

10. Benegal V, Veleyudhan A, Jain S. The social cost of alcholism (Karnataka). NIMHANS Journal 2000;18.

11. Gururaj G NG, Benegal V. Alcohol control series 1: Burden and socioeconomic impact of alcohol- The Bangalore Study. 2006. http://www. searo.who.int/entity/mental_health/documents/9290222727.pdf

12. Sarkar NN. Barriers to condom use. Eur J Contracept Reprod Health Care 2008;13:114-22.

13. Nirmala M, George R, Benegal V. Children of alcoholics: the different dimensions. NIMHANS De-addiction Quarterly, 2000:5.

14. Bloomberg Markets. USD INR: Bloomberg. https://www.bloomberg. com/quote/USDINR:CUR

15. Centers for Disease Control and Prevention Office of the Associate Director for Policy. Definition of policy Atlanta, GA. 2015. https:// www.cdc.gov/policy/analysis/process/definition.html

16. Prasad R. Alcohol use on the rise in India. The Lancet 2009;373:17-18.

17. Jernigan D. Global developments in alcohol policies: Progress in implementation of the WHO global strategy to reduce the harmful 
use of alcohol since 2010. 2017. http://www.who.int/substance abuse/activities/fadab/msb_adab_gas_progress_report.pdf?ua $=1$

18. Arksey H, O'Malley L. Scoping studies: towards a methodological framework. Int J Soc Res Methodol 2005;8:19-32.

19. Levac D, Colquhoun H, O'Brien KK. Scoping studies: advancing the methodology. Implement Sci 2010;5:69.

20. Pillai A, Nayak MB, Greenfield TK, et al. Patterns of alcohol use, their correlates, and impact in male drinkers: a population-based survey from Goa, India. Soc Psychiatry Psychiatr Epidemiol 2013:48:275-82.

21. Nadkarni $A$, Bhatia $U$, Velleman $R$, et al. Supporting addictions affected families effectively (SAFE): a mixed methods exploratory study of the 5-step method delivered in Goa, India, by lay counsellors. Drugs: Education, Prevention and Policy 2017.
22. Nadkarni A, Weiss HA, Naik A, et al. The six-year outcome of alcohol use disorders in men: A population based study from India. Drug Alcohol Depend 2016;162:107-15.

23. Nadkarni A, Weobong B, Weiss HA, et al. Counselling for Alcohol Problems (CAP), a lay counsellor-delivered brief psychological treatment for harmful drinking in men, in primary care in India: a randomised controlled trial. The Lancet 2017;389:186-95.

24. Liberati A, Altman DG, Tetzlaff J, et al. The PRISMA statement for reporting systematic reviews and meta-analyses of studies that evaluate health care interventions: explanation and elaboration. Ann Intern Med 2009;151:W.

25. World Health Organization. Global strategy to reduce the harmful use of alcohol. 2010. http://apps.who.int/iris/bitstream/handle/10665/ 44395/9789241599931_eng.pdf?sequence=1 